\title{
Protease Inhibitor and Metabolic Alteration
}

\author{
Inhibidor de la Proteasa y Alteraciones Metabólicas \\ Cavenaghi, F. M.; Bataglion, C. A. N.; Paula, P. C.; Motta, A. C. F. \& Komesu, M. C.
}

CAVENAGHI, F. M.; BATAgliON, C. A. N.; PAUla, P. C.; MOTTA, A. C. F. \& KOMESU, M. C. Protease inhibitor and metabolic alteration. Int. J. Morphol., 30(2):439-444, 2012.

SUMMARY: Protease inhibitors (PIs), part of HAART (Highly Active Antiretroviral Therap) are selective, competitive inhibitors of protease, a crucial enzyme to viral maturation, infection and replication. A lipodystrophic syndrome has been reported in individuals treated with HAART, and associated to hyperglycemia, hypercholesterolemia, hypertrigliceridemia, hyperlipidemia, hypertension and hypreinsulinemia. The HAART-associated metabolic abnormalities were first associated with protease inhibitors, Ritonavir mostly, but the mechamisns that underlie these metabolic alterations are to date, not completely understood. Since PIs are candidate to be the drug of choice for other diseases treatment, such as the Hepatitis C, malaria and some types of cancer, it seems to be important to clarify the metabolic alterations associated to PIs. Wistar rats were treated twice a week with $30 \mathrm{mg} / \mathrm{kg}$ Ritonavir for 4 and 8 weeks. Total cholesterol, HDL, LDL, VLDL, triglycerides and glycemic levels were measured by the end of each period of time selected. To avoid confunding effects of food intake, the animals were fasted 16 hours before. Our results showed rapid increase in serum triglycerides, total cholesterol, LDL-C and glycemic levels. No significant differences were observed for HDL-C or VLDL serum levels. Our study addresses the importance to observe the possible family history of dyslipidemia or diabetes, and control any other cardiovascular and diabetes risk factors when using protease inhibitors

KEY WORDS: Protease inhibitors; Dyslipidemia; Metabolic disorders.

\section{INTRODUCTION}

The Highly Active Antiretroviral Therapy (HAART), also known as ART (antiretroviral therapy), is available since 1996 and uses three or four different antiretrovirals, in general two nucleoside analogue reverse transcriptase inhibitors (NRTIs) + one or two protese inhibitors (PIs), or the non-nucleoside reverse transcriptase inhibitors (NNRTIs) and cell membrane fusion inhibitors (Lenhard et al., 2000; Wynn et al., 2004).

HAART is the treatment of choice for established HIV-infection and for post-exposure prophylaxis. HAART had a dramatic impact on the morbidity and mortality of individuals living with human immunodeficiency virus (HIV). However, strict adherence to the HAART is imperative to limit the development of viral resistance, and noxious side effects, adverse drug reactions and drug interactions may lead to nonadherence to HAART, placing the patient at risk for treatment failure. Studies have shown up to $70 \%$ of HIV patiens are intentionally nonadherent to treatment (Wynn et al.).

Protease inhibitors (PIs) are selective, competitive inhibitors of protease, a crucial enzyme to viral maturation, infection and replication. They are the largest class of drugs against HIV, acting at a very late stage in the HIV replication process. HIV protease acts right before the viral proteins leave the cell. However, the ability of these agents to suppress virus replication is limited, and the selection of drug resistant HIV variants is not uncommon(Patick \& Potts, 1998). Also, the HIV protease is a member of the aspartyl-protease enzyme family, which includes renin and pepsin, and side effects of PIs may include gastrointestinal disruptions, dysfunction of lipids and glucose metabolism, sexual dysfunction, hepatic toxicity, and increased risk of bleeding (Wynn et al.).

All PIs are inhibitors of metabolism of cytochrome P450 system, specifically at the 3A4 enzyme (CYP3A4). Cytochrome P4503A is involved in the metabolic clearance of approximately $50 \%$ of drugs in the market, and accounts for about $70 \%$ of the cytochrome $\mathrm{P} 450$ content in the small intestine. Patients with inhibition of CYP system may be at risk for drug interactions and metabolic complications (Kalitsky-Szirtes et al., 2004). 
Ritonavir is "the pan-inhibitor" or "multiple P450 inhibitor". The concurrent use with other agents which are metabolized by CYP3A4, such as different analgesics, antiarrythmic agents, antibiotics, anticoagulants, anticonvulsivants, antiemetics, and antifungal agents, may result in increased levels of these drugs in plasma, and a potential risk for adversal effects (Wynn et al.). Also, it seems like PIs may inhibit GLUT-4, the predominant glucose transporter in muscle and fat, and that alterations in GLUT4 expression may alter insulin sensitivity (Hruz et al., 2011).

A lipodystrophic syndrome has been reported in individuals on HAART, and includes fat redistribution with peripheral lipoatrophy, central or visceral lipohypertrophy, associated to hyperglycemia, hypercholesterolemia, hypertrigliceridemia, hyperlipidemia, hypertension and hypreinsulinemia (Leow et al., 2003). The prevalence of lipodistrophy in patients with HAART is approximately $40 \%$, and levels are differentially affected by anti retrovirals. The HAART-associated metabolic abnormalities were first associated with PIs, Ritonavir mostly, but other antivirals have also been implicated (Kinlaw \& Marsh, 2004).

Serum lipid abnormalities seem to occur shortly after HAART beguining therapy (weeks to months) depending on the drug choice and dose, but the pathogenic pathways of PI-associated dyslipidemia is not completely understood (Calza et al., 2003).

Drug therapy for dyslipidemia in persons receiving HAART may be problematic, since many statins (3Hydroxy-3-Methylglutaryl coenzyme A or HMG-Co A reductase inhibitors), are extensively metabolized by CYP3A4. Overexposure to statins may significantly increase risk for liver and skeletal muscle toxicity (Calza et al.).

\section{MATERIAL AND METHOD}

Ritonavir is a prototype of PI, and causes the most severe metabolic abnormalities in humans and animal models. Lypodistrophy syndrome may occur in up to $50 \%$ taking PI (Temple et al., 2003). Ritonavir (Norvirx solution) has been used in experimental studies $(10 \mathrm{mg} / \mathrm{kg} /$ dose for Sprague-Dawley rats and Rhesus monkeys, and $45-60 \mathrm{mg} /$ $\mathrm{kg} / \mathrm{d}, 50 \mathrm{ml}$ or $2 \mathrm{mg} /$ day for mice). It was well absorbed, 80 $100 \%$ in both animal species. The use of ritonavir for two weeks caused a elevation of plasma triglycerides and alterations on glycemic levels was observed some time after (Lenhard et al.; Xu et al., 2004).

Our animals (Wistar rats) were treated twice a week with $30 \mathrm{mg} / \mathrm{kg}$ Ritonavir for 4 and 8 weeks. To determine the effects of the medication on in vivo lipoproteins, triglycerides, total cholesterol, HDL, LDL and VLDL were measured by the end of each period of time selected. To avoid confounding effects of food intake, the animals were fasted 16 hours before the blood collection. Blood samples were collected in the morning, from rats under anesthesia. Glycemia were evaluated by the end of the period of the time selected for the study, by measuring serum glycemic levels.

\section{RESULTS}

Data about cholesterol, triglycerides and glycemia are presented in Tables I and II.

\section{DISCUSSION}

The complexity about the effects of HIV infections and HAART regimens, associated with the effects of other related comorbidities, has challenged the studies of adverse effects in HIV-infection treatment (Hruz et al.).

HAART, and mostly PIs, have been associated with lipid disorders, such as hypertriglyceridemia (Friis-Møller et al., 2003; Noor et al., 2004; Dubé et al., 2008), lipodistrophy (Carr et al., 1999), increased total cholesterol and LDL-C serum levels, and lower HDL-C serum levels (Friis-Møller et al.). Longitudinal study with HIV infected patients suggests that there are decreases in total cholesterol, HDL-C and LDL-C at the time of infection, before treatment, and with initiation of HAART, total and LDL cholesterol increases but HDL-C low levels persists (Grinspoon \& Carr, 2005). Studies indicated that some protease inhibitor (PIs), mostly Ritonavir, may increase hepatic triglycerides synthesis and plasma triglyceride levels (Lenhard et al.). Some protease inhibitors (PIs) also tend to increase total cholesterol serum levels, but this effect varies among different individual PIs (Périard et al., 1999).

Our results showed rapid increase in serum triglycerides (Table I), but tendency to come back to normal levels with prolonged use ( 8 weeks), increased LDL-C levels (Table I), and increased total cholesterol serum levels soon after the initial use of PI. No significant differences were observed for HDL-C or VLDL serum levels.

Impaired insulin uptake is associated with PIs use (Noor et al., 2004) but there are controversies (Dubé et al.). It is believed that ritonavir and liponavir induce insulin resistance at therapeutic concentrations, mostly by direct inhibition of GLUT4 but also modulating insulin sensitivity 
Table I. Serum triglycerides, total cholesterol and fractions in animals control and treated with protease inhibitor.

\begin{tabular}{|c|c|c|c|c|}
\hline & \multicolumn{2}{|c|}{ Control } & \multicolumn{2}{|c|}{ Trated } \\
\hline & 4 weeks & 8 weeks & 4 weeks & 8 weeks \\
\hline \multirow[t]{6}{*}{ Triglycerides } & 47.58 & 63.77 & 76.98 & 67.58 \\
\hline & 64.32 & 78.30 & 92.45 & 74.01 \\
\hline & 36.12 & 52.83 & 101.32 & 65.20 \\
\hline & 66.08 & 61.89 & 74.53 & 76.65 \\
\hline & 86.34 & 71.13 & 98.11 & 89.87 \\
\hline & $60.08 \pm 19.19$ & $65.58 \pm 9.64$ & $88.67 \pm 12.24$ & $74.66 \pm 9.68$ \\
\hline \multirow[t]{2}{*}{ Mann Whitney U Test } & & & $\mathrm{U}$ canc. $=2 \mathrm{P}[\mathrm{U}]=0.016$ & $\mathrm{U}$ canc. $=6 \mathrm{P}[\mathrm{U}]=0.111$ \\
\hline & & & $\uparrow$ & \\
\hline \multirow[t]{8}{*}{ Total Cholesterol } & 60.12 & 80.25 & 78.89 & 100.43 \\
\hline & 63.19 & 90.30 & 70.37 & 97.91 \\
\hline & 61.53 & 92.91 & 75.93 & 92.64 \\
\hline & 60.92 & 90.49 & 69.75 & 104.91 \\
\hline & 67.73 & 85.19 & 69.88 & 94.48 \\
\hline & $62.69 \pm 3.03$ & $89.82 \pm 7.17$ & $72.96 \pm 4.19$ & $96.07 \pm 6.67$ \\
\hline & & & $\mathrm{U}$ canc. $=0 \mathrm{P}[\mathrm{U}]=0.004$ & $\mathrm{U}$ canc. $=1 \mathrm{P}[\mathrm{U}]=0.008$ \\
\hline & & & $\uparrow$ & $\uparrow$ \\
\hline \multirow[t]{7}{*}{ HDL-C } & 40.84 & 37.33 & 52.27 & 52.16 \\
\hline & 43.34 & 43.20 & 36.27 & 41.95 \\
\hline & 37.35 & 44.67 & 49.20 & 48.78 \\
\hline & 38.47 & 38.27 & 35.73 & 48.22 \\
\hline & 43.41 & 45.60 & 37.73 & 45.02 \\
\hline & $40.68 \pm 2.76$ & $41.81 \pm 377$ & $42.24 \pm 7.86$ & $47.21 \pm 3.88$ \\
\hline & & & $\mathrm{U}$ canc. $=11 \mathrm{P}[\mathrm{U}]=0.421$ & $\mathrm{U}$ canc. $=5 \mathrm{P}[\mathrm{U}]=0.075$ \\
\hline \multirow[t]{8}{*}{ LDL-C } & 9.79 & 30.17 & 11.23 & 38.76 \\
\hline & 6.99 & 37.44 & 15.61 & 31.16 \\
\hline & 16.96 & 41.68 & 16.47 & 37.50 \\
\hline & 10.14 & 39.85 & 19.12 & 41.36 \\
\hline & 7.06 & 23.37 & 12.53 & 31.49 \\
\hline & $10.17 \pm 4.08$ & $34.50 \pm 3.77$ & $14.99 \pm 4.75$ & $36.05 \pm 4.53$ \\
\hline & & & $\mathrm{U}$ canc. $=4 \mathrm{P}[\mathrm{U}]=0.048$ & $\mathrm{U}$ canc. $=4 \mathrm{P}[\mathrm{U}]=0.048$ \\
\hline & & & $\uparrow$ & $\uparrow$ \\
\hline \multirow[t]{7}{*}{ VLDL } & 9.51 & 12.75 & 15.39 & 9.51 \\
\hline & 12.86 & 15.66 & 18.49 & 14.80 \\
\hline & 7.22 & 10.56 & 10.26 & 13.04 \\
\hline & 13.21 & 12.37 & 14.90 & 15.33 \\
\hline & 17.26 & 16.22 & 19.62 & 17.97 \\
\hline & $12.01 \pm 3.83$ & $13.51 \pm 2.92$ & $15.73 \pm 3.65$ & $14.13 \pm 3.12$ \\
\hline & & & $\mathrm{U}$ canc. $=7 \mathrm{P}[\mathrm{U}]=0.155$ & $\mathrm{U}$ canc. $=11 \mathrm{P}[\mathrm{U}]=0.421$ \\
\hline
\end{tabular}

via other insulin signaling pathways, including IRS-1 and AKT (Yan \& Hruz, 2005; Hurz et al.). Our results showed increased glycemic levels in treated animals. The mechanisms involved deserves additional studies. 
Table II. Glycaemia in animals control and treated with protease inhibitor.

\begin{tabular}{ccccc}
\hline & \multicolumn{2}{c}{ Control } & \multicolumn{2}{c}{ Trated } \\
\cline { 2 - 5 } Glycaemia & 4 weeks & 8 weeks & 4 weeks & 8 weeks \\
& 72.41 & 108.87 & 72.59 & 129.65 \\
66.45 & 113.09 & 78.96 & 144.37 \\
69.38 & 118.33 & 77.52 & 120.26 \\
62.87 & 98.39 & 80.46 & 105.79 \\
74.92 & 102.89 & 70.59 & 141.48 \\
& $\mathbf{6 9 . 2 8} \pm \mathbf{4 . 7 6}$ & $\mathbf{1 0 8 . 3 1 \pm 7 . 9 2}$ & $\mathbf{7 6 . 0 2 \pm 4 . 2 3}$ & $\mathbf{1 2 8 . 3 1 \pm 1 5 . 0 5}$ \\
\hline Mann Whitney U Test & & U canc. $=\mathbf{3}$ P[U] $\mathbf{= 0 . 0 0 2 8}$ & U canc. $=\mathbf{3 ~ P [ U ] = 0 . 0 2 8}$
\end{tabular}

Dyslipidemia and hyperglycemia may be observed in HIV-positive patients not on HAART, and dyslipidemia may be caused by the HIV infection, as well as any other infectious disease (Baza Caraciolo et al., 2007; Estrada \& Portilla, 2011). However, among HIV-infected adults with HAART metabolic alterations are more prevalent than in HIV-infected not on HARRT (Grinspoon \& Carr). Our study with Ritonavir showed higher triglyceride, total cholesterol, LDL-C and glycose serum levels in animals not HIV-infected and without previous induction of any metabolic alterations.

Originally used as monotherapy, Ritonavir is now used as enhancer for other PIs (Hull \& Montaner, 2011). Some PIs may also be used in the Hepatitis C treatment (Gordon et al., 2011; Poordad et al., 2011) as anti-protozoals, in malaria and other gastrointestinal infections (Dunn et al.,
2007). There are also studies investigating whether PIs could be used as a cancer therapy (Gills et al., 2007).

In any case, our study addresses that it is important to observe the possible family history of dyslipidemia or diabetes, control any other cardiovascular and diabetes risk factors when using PIs. Ritonavir is a potent inhibitor of CYP3A4 and of CHP2D6, mostly when used in a dose of $600 \mathrm{mg}$ twice daily. However, studies have shown that even low-dose (100mg twice daily) may cause some collateral underisable effects (Aarnoutse et al., 2005; Grinspon \& Carr).

ACKNOWLEDGMENTS. The authors thanks Prof. Dr. Miguel Angel Sala di Matteo for the Spanish translation.

CAVEnaghi, F. M.; BAtaglion, C. A. N.; PAUla, P. C.; MOTTA, A. C. F. \& KOMESU, M. C. Inhibidor de la proteasa y alteraciones metabólicas. Int. J. Morphol., 30(2):439-444, 2012.

RESUMEN: Los inhibidores de la proteasa (IP), que forman parte de la terapia HAART (terapia antirretroviral altamente activa), son inhibidores selectivos y competitivos de la proteasa, enzima crucial para la maduración, infección y replicación viral. Un síndrome lipodistrófico, asociado a hiperglucemia, hipercolesterolemia, hipertrigliceridemia, hiperlipidemia, hipertensión e hiperinsulinemia, ha sido relatado en pacientes tratados con HAART. Las anomalías metabólicas asociadas a la HAART fueron relacionadas, inicialmente, a los inhibidores de la proteasa, principalmente el Ritonavir, pero los mecanismos que relacionados a estas alteraciones metabólicas son poco comprendidos. Dado que los IP son posibles candidatos a fármacos de elección para tratamiento de otras enfermedades, como hepatitis C, malaria y algunos tipos de cáncer, es importante esclarecer las alteraciones metabólicas asociadas a los inhibidores de la proteasa. Ratas Wistar fueron tratadas dos veces por semana con $30 \mathrm{mg} / \mathrm{kg}$ de Ritonavir por 4 y 8 semanas. Fueron determinados los niveles de colesterol total, HDL, LDL, VLDL, triglicéridos y glucemia, al final de cada período considerado. Para evitar la interferencia de la ingestión de alimentos en las determinaciones de laboratorio, los animales fueron sometidos a un ayuno previo de 16 horas. Nuestros resultados mostraron un rápido aumento sérico de los niveles de triglicéridos, colesterol total, LDL-C y glucemia. No se observaron diferencias significativas para los niveles séricos de HDL-C o VLDL. Nuestro estudio apunta a la importancia de considerar los posibles antecedentes familiares de dislipidemia o diabetes, y controlar cualquier otro factor de riesgo cardiovascular y de diabetes cuando se utilizan los inhibidores de la proteasa.

PALABRAS CLAVE: Inhibidores de la proteasa; Dislipidemia; Trastornos metabólicos. 


\section{REFERENCES}

Aarnoutse, R. E.; Kleinnijenhuis, J.; Koopmans, P. P.; Touw, D. J.; Wieling, J.; Hekster, Y. A. \& Burger, D. M. Effect of low-dose ritonavir (100 mg twice daily) on the activity of cytochrome P450 2D6 in healthy volunteers. Clin. Pharmacol. Ther., 78(6):664-74, 2005.

Baza Caraciolo, B.; Pèrez de Oteyza, C.; Carró Montiel, D.; Carrió Montiel, J. C.; Salguero Aparicio, M. \& Del Romero Guerrero, J. Lipid profile in untreated HIV positive patients. HIV infection: cardiovascular risk factor?. An. Med. Interna, 24(4):160-7, 2007.

Calza, L.; Manfredi, R. \& Chiodo, F. Dyslipidaemia associated with antiretroviral therapy in HIV-infected patients. $J$. Antimicrob. Chemother., 53(1):10-4, 2004.

Carr, A.; Samaras, K.; Thorisdottir, A.; Kaufmann, G. R.; Chisholm, D. J. \& Cooper, D. A. Diagnosis, prediction, and natural course of HIV-1 protease-inhibitor-associated lipodystrophy, hyperlipidaemia, and diabetes mellitus: a cohort study. Lancet, 353(9170):2093-9, 1999.

Dubé, M. P.; Shen, C.; Greenwald, M. \& Mather, K. J. No impairment of endothelial function or insulin sensitivity with 4 weeks of the HIV protease inhibitors atazanavir or lopinavir-ritonavir in healthy subjects without HIV infection: a placebo-controlled trial. Clin. Infect. Dis., 47(4):567-74, 2008 .

Dunn, L. A.; Andrews, K. T.; Mccarthy, J. S.; Wright, J. M.; SkinnerAdams, T. S.; Upcroft, P. \& Upcroft, J. A. The activity of protease inhibitors against Giardia duodenalis and metronidazole-resistant Trichomonas vaginalis. Int. J. Antimicrob. Agents, 29(1):98-102, 2007.

Estrada, V. \& Portilla, J. Dyslipidemia related to antiretroviral therapy. AIDS Rev., 13(1):49-56, 2011.

Friis-Møller, N.; Weber, R.; Reiss, P.; Thiébaut, R.; Kirk, O.; D'arminio Monforte, A.; Pradier, C.; Morfeldt, L.; Mateu, S.; Law, M.; El-Sadr, W.; De Wit, S.; Sabin, C. A.; Phillips, A. N.; Lundgren, J. D. \& DAD Study Group. Cardiovascular disease risk factors in HIV patients--association with antiretroviral therapy. Results from the DAD study. AIDS, 17(8):1179-93, 2003.

Gills, J. J.; Lopiccolo, J.; Tsurutani, J.; Shoemaker, R. H.; Best, C. J.; Abu-Asab, M. S.; Borojerdi, J. Warfel, N. A.; Gardner, E. R.; Danish, M.; Hollander, M. C.; Kawabata, S.; Tsokos, M.; Figg, W. D.; Steeg, P. S. \& Dennis, P. A. Nelfinavir, A lead HIV protease inhibitor, is a broad-spectrum, anticancer agent that induces endoplasmic reticulum stress, autophagy, and apoptosis in vitro and in vivo. Clin. Cancer Res., 13(17):518394, 2007.
Gordon, S.; Bacon, B.; Lawitz, E.; Marcellin, P.; Vierling, J.; Zeuzem, S.; Poordad, F.; Albrecht, J. \& Esteban, R. HCV RESPOND-2 Final Results: High Sustained Virologic Response among Genotype-1 Previous Non-responders and Relapsers to pegIFN/RBV when Re-treated with BOC + PEGINTRON/RBV. 18th Conference on Retroviruses and Opportunistic Infections (CROI 2011), February 27-March 2, Boston, 2011.

Grinspoon, S. \& Carr, A. Cardiovascular risk and body-fat abnormalities in HIV-infected adults. N. Engl. J. Med., 352(1):48-62, 2005.

Hruz, P. W.; Yan, Q.; Tsai, L.; Koster, J.; Xu, L.; Cihlar, T. \& Callebaut, C. GS-8374, a novel HIV protease inhibitor, does not alter glucose homeostasis in cultured adipocytes or in a healthy-rodent model system. Antimicrob. Agents Chemother., 55(4):1377-82, 2011.

Hull, M. W. \& Montaner, J. S. Ritonavir-boosted protease inhibitors in HIV therapy. Ann. Med., 43(5):375-88, 2011.

Kalitsky-Szirtes, J.; Shayeganpour, A.; Brocks, D. R. \& PiquetteMiller, M. Suppression of drug-metabolizing enzymes and efflux transporters in the intestine of endotoxin-treated rats. Drug Metab. Dispos., 32(1):20-7, 2004.

Kinlaw, W. B. \& Marsh, B. Adiponectin and HIV-lipodystrophy: taking HAART. Endocrinology, 145(2):484-6, 2004.

Leow, M. K.; Addy, C. L. \& Mantzoros, C. S. Clinical review 159: Human immunodeficiency virus/highly active antiretroviral therapy-associated metabolic syndrome: clinical presentation, pathophysiology, and therapeutic strategies. J. Clin. Endocrinol. Metab., 88(5):1961-76, 2003.

Lenhard, J. M.; Croom, D. K.; Weiel, J. E. \& Winegar, D. A. HIV protease inhibitors stimulate hepatic triglyceride synthesis. Arterioscler. Thromb. Vasc. Biol., 20(12):2625-9, 2000.

Noor, M. A.; Parker, R. A.; O'Mara, E.; Grasela, D. M.; Currie, A.; Hodder, S. L.; Fiedorek, F. T. \& Haas, D. W. The effects of HIV protease inhibitors atazanavir and lopinavir/ritonavir on insulin sensitivity in HIV-seronegative healthy adults. AIDS, 18(16):2137-44, 2004.

Patick, A. K. \& Potts, K. E. Protease inhibitors as antiviral agents. Clin. Microbiol. Rev., 11(4):614-27, 1998.

Périard, D.; Telenti, A.; Sudre, P.; Cheseaux, J. J.; Halfon, P.; Reymond, M. J.; Marcovina, S.; Glauser, M. P.; Nicod, P.; Darioli, R. \& Mooser, V. Atherogenic dyslipidemia in HIVinfected individuals treated with protease inhibitors. The Swiss HIV Cohort Study. Circulation, 100(7):700-5, 1999. 
Poordad, F.; Mccone, J. Jr.; Bacon, B. R.; Bruno, S.; Manns, M. P.; Sulkowski, M. S.; Jacobson, I. M.; Reddy, K. R.;Goodman, Z. D.; Boparai, N.; Dinubile, M. J.; Sniukiene, V.; Brass, C. A.; Albrecht, J. K.; Bronowicki, J. P. \& SPRINT-2 Investigators. Boceprevir for untreated chronic HCV genotype 1 infection. N. Engl. J. Med., 364(13):1195-206, 2011.

Temple, M. E.; Koranyi, K. I. \& Nahata, M. C. Lipodystrophy in HIV-infected pediatric patients receiving protease inhibitors. Ann. Pharmacother., 37(9):1214-8, 2003.

Xu, A.; Yin, S.; Wong, L. C.; Chan, K. W. \& Lam, K. S. L. Adiponectin ameliorates dyslipidemia induced by the human immunodeficiency virus protease inhibitor ritonavir in mice. Endocrinology, 145(2):487-94, 2004.

Yan, Q. \& Hruz, P. Direct comparison of the acute in vivo effects of HIV protease inhibitors on peripheral glucose disposal. $J$. Acquir. Immune Defic. Syndr., 40(4):398-403, 2005.

Wynn, G. H.; Zapor, M. J.; Smith, B. H.; Wortmann, G.; Oesterheld, J. R.; Armstrong, S. C. \& Cozza, K. L. Antiretrovirals, part 1: overview, history, and focus on protease inhibitors. Psychosomatics, 45(3):262-70, 2004.
Correspondence to: Marilena Chinali Komesu

Faculdade de Odontologia de Ribeirão Preto - USP

Departamento de Morfologia, Estomatologia e Fisiologia

Avenida do Café, s/no, Monte Alegre, 14.040-904

Ribeirão Preto, São Paulo

BRAZIL

Phone: (55) 1636023985

Email: mckomesu@usp.br

Received: 30-08-2011

Accepted: 12-02-2012 